\title{
Puerarin attenuates glucocorticoid-induced apoptosis of hFOB1.19 cells through the JNK- and Akt-mediated mitochondrial apoptotic pathways
}

\author{
DONGDONG YU, SHUAI MU, DANYANG ZHAO, GUANGBIN WANG, \\ ZHIGUANG CHEN, HONGFEI REN and QIN FU
}

Department of Orthopedic Surgery, The Shengjing Hospital of China Medical University, Liaoning 110004, P.R. China

Received November 11, 2014; Accepted May 21, 2015

DOI: $10.3892 /$ ijmm.2015.2258

\begin{abstract}
Puerarin is an active component of Pueraria lobata, which is a commonly used Chinese herbal medicine for the treatment of osteoporosis. The present study aimed to evaluate the osteoprotective effect of puerarin on glucocorticoid (GC)-induced apoptosis of osteoblasts in vitro. The effects of puerarin on dexamethasone (DEX)-induced cell apoptosis were assessed using enzyme-linked immunosorbent assay and a terminal deoxynucleotidyl transferase dUTP nick-end labeling assay, and found that the viability of hFOB1.19 cells was significantly increased following exposure to between $10^{-6}$ and $10^{-10} \mathrm{M}$ puerarin, with a maximal anti-apoptotic effect at a concentration of $10^{-8} \mathrm{M}$. In addition, compared with the control group, puerarin upregulated the transcription and protein levels of B-cell lymphoma-2 and downregulated B-cell-associated X protein in the hFOB1.19 cells. Puerarin attenuated the DEX-induced release of cytochrome $c$ and cleavage of caspase-3, and treatment with puerarin inhibited the c-Jun N-terminal kinase (JNK) pathway and activated the phosphoinositide 3-kinase (PI3K)/Akt pathway in the hFOB1.19 cells. Furthermore, the Akt inhibitor, LY294002, partly eliminated the protective effect of puerarin on DEX-induced apoptosis, and puerarin combined with the JNK inhibitor, SP600125, suppressed DEX-induced apoptosis to a lesser extent than in the cells treated with SP600125 alone. These results suggested that the JNK and PI3K/Akt signaling pathways mediate the inhibitory effects of puerarin on apoptosis in the hFOB1.19 cells. In conclusion, puerarin prevented DEX-induced apoptosis of hFOB1.19 cells via inhibition of the JNK pathway and activation of the PI3K/Akt signaling pathway in the cells, dependent on the mitochondrial apoptotic
\end{abstract}

Correspondence to: Dr Qin Fu, Department of Orthopedic Surgery, The Shengjing Hospital of China Medical University, 36 Sanhao Street, Liaoning 110004, P.R. China

E-mail: qinfucmu@163.com

Key words: puerarin, apoptosis, osteoporosis, AKT, c-Jun N-terminal kinase pathway. These results support puerarin as a promising target in the treatment of GC-induced osteoporosis.

\section{Introduction}

Glucocorticoids (GCs) are widely used in the treatment of a variety of diseases, including autoimmune diseases, bronchial asthma and skin diseases. Although GCs are potent antiinflammatory agents, long-term use can lead to various adverse effects, including osteoporosis (3). GC-induced osteoporosis (GIOP), the major cause of which is considered to be impairment of bone formation $(1,2)$ is the most common form of secondary osteoporosis (3). Excess GCs inhibits osteoblast maturation and differentiation, and promotes the apoptosis of osteoblasts (4), which is considered to be the third major cause of GIOP (4). Therefore, anti-apoptosis of osteoblasts is generally served as the pivotal target for the prevention of GCs-induced osteoporosis.

Puerarin, a C-glycoside compound, is a major component derived from Pueraria lobata (Willd.), which is a commonly used Chinese herbal medicine (5). A number of investigations have been performed internationally to identify the pharmacology functions of puerarin. Previous studies have reported that puerarin possesses antioxidant (6,7), anti-inflammatory (8) and anti-apoptotic properties (7). In addition, investigations have revealed that puerarin has the ability to resist apoptosis in a variety of cells, including liver cells (9), osteoblasts (10), kidney cells (11) and nerve cells (12). Although it acts as an important regulator of cell death, the role of puerarin in the GC-induced apoptosis of osteoblasts and the underlying mechanisms remain to be fully elucidated.

Apoptosis is an essential process in maintaining homeostasis under normal conditions (13). To date, studies have indicated that there are two predominant apoptotic pathways: the extrinsic, or death receptor, pathway and the intrinsic, or mitochondrial, pathway (14), B-cell-associated X protein (Bax) and B-cell lymphoma (Bcl)-2 are members of the Bcl-2 family, which is closely associated with the mitochondrial apoptotic pathway (15). Bcl-2 protein forms heterodimer complexes with Bax proteins, leading to the release of cytochrome $c$ from the mitochondria and the induction of apoptosis (16). Liu et al (17) found that puerarin suppresses the apoptosis of human osteo- 
blasts by increasing the protein levels of Bcl-2, while decreasing the levels of Bax in a dose-dependent manner. Wang et al (18) reported that puerarin prevents MPP-induced apoptosis of PC12 cells by suppressing the release of mitochondrial cytochrome $c$ and activation of caspase-3. Several signaling pathways, including the phosphoinositide 3-kinase (PI3K)/Akt pathway and c-Jun N-terminal kinase (JNK) pathway, have also been demonstrated to regulate the apoptosis of osteoblastic cells (18-22). It has been observed that puerarin inhibits serum-free-induced apoptosis of human osteoblast cells (23), however, whether puerarin has an effect on the GC-induced apoptosis of osteoblast remains to be elucidated.

The present study aimed to investigate the effects of puerarin on dexamethasone (DEX)-induced apoptosis in hFBO1.19 cells and examine the expression of several apoptosis-associated proteins, including Bcl-2, Bax, caspase- 3 and cytochrome $c$ were examined, to determine whether the effects were mediated by the JNK and PI3K/Akt signaling pathways.

\section{Materials and methods}

Reagents. DEX and 17 $\beta$-estradiol (E2) were purchased from Sigma-Aldrich (St. Louis, MO, USA). Puerarin (dissolved in dimethyl sulfoxide (DMSO; molecular weight 416.38; purity $>98 \%$ ) was obtained from the National Institute for the Control of Pharmaceutical and Biological Products (Beijing, China). DEX and pueranrin were used in a concentration gradient between $10^{-5}$ and $10^{-10} \mathrm{M}$. The final concentration of DEX $\left(10^{-5} \mathrm{M}\right)$ and of E2 $\left(10^{-5} \mathrm{M}\right)$ was dissolved in ethanol, and puerarin $\left(10^{-5} \mathrm{M}\right)$ was dissolved in DMSO (Sigma-Aldrich). The DEX, puerarin and E2 were stored at $-20^{\circ} \mathrm{C}$.

Cell culture. The conditionally immortalized human fetal osteoblastic cell line, hFOB1.19, was provided by Dr Meng (China Medical University, Shenyang, China). The cells were cultured, according to the procedures of American Type Culture Collection (Manassas, VA, USA) and those previously described (24). For in vitro proliferation, the hFOB1.19 cells were maintained in non-differentiation medium consisting of 1:1 Dulbecco's modified Eagle's medium (DMEM)/F-12 (GE Healthcare Life Sciences, Logan, UT, USA) medium with $10 \%$ fetal bovine serum (FBS; Biological Industries, Beit-Haemek, Israel), and $0.3 \mathrm{~g} / \mathrm{l} \mathrm{G} 418$ (Sigma-Aldrich), and were cultured in a humidified incubator with $5 \% \mathrm{CO}_{2}$ at $33.4^{\circ} \mathrm{C}$. The medium was replaced twice a week and the cells were cultured using $0.25 \%$ trypsin with $0.02 \%$ ethylenediaminetetraacetic acid (Sigma-Aldrich).

Cell proliferation assay. The hFOB1.19 cells, at $80-90 \%$ confluency, were inoculated at $3 \times 10^{3}$ cells/well in 96-well plates and the number of cells were quantified using an MTS assay, according to the manufacturer's instructions (Promega Corporation, Madison, WI, USA). Briefly, $20 \mu \mathrm{l}$ MTS solution was added to $100 \mu \mathrm{l}$ culture medium in each well. Following incubation for $2 \mathrm{~h}$ at $37^{\circ} \mathrm{C}$, the absorbance was read at a wavelength of $490 \mathrm{~nm}$ on a microplate reader (Varioskan LUX; Thermo Fisher Scientific, Vantaa, Finland), with measurements presented as the mean of at least three independent experiments, with each data point based upon three replicates.

To assess the effects of puerarin on cell proliferation, the cells $\left(5 \times 10^{3}\right.$ per well) were incubated in conditioned medium at $37^{\circ} \mathrm{C}$ for 3,12 and $24 \mathrm{~h}$ at a concentration gradient between $0,10^{-5}$ and $10^{-9} \mathrm{M}$. Cells were incubated with DEX in conditioned medium for 24,48 and $72 \mathrm{~h}$ at a concentration gradient between $0,10^{-5}$ and $10^{-10} \mathrm{M}$.

Determination of cell death. Enzyme-linked immunosorbent assay (ELISA) detection was performed to detect the levels of apoptosis, as previously described (25). According to the kit protocol (Roche Molecular Biochemicals, Mannheim, Germany), the cells were plated at a density of $1 \times 10^{4}$ cells/ well in 24 -well plates at $37^{\circ} \mathrm{C}$ for 1 day and then cultured in the absence or presence of puerarin $\left(10^{-6}-10^{-9} \mathrm{M}\right)$ and E2 $\left(10^{-5} \mathrm{M}\right)$ at $37^{\circ} \mathrm{C}$ for $3 \mathrm{~h}$, followed by incubation with $10^{-5} \mathrm{M}$ DEX medium at $37^{\circ} \mathrm{C}$ for $48 \mathrm{~h}$. The cells were then rinsed with phosphate-buffered saline (PBS) and incubated with $0.5 \mathrm{ml}$ lysis buffer (Beyotime Institute of Biotechnology, Shanghai, China) at $4^{\circ} \mathrm{C}$ for $30 \mathrm{~min}$, and centrifuged at $4^{\circ} \mathrm{C}$ for $10 \mathrm{~min}$ at $200 \mathrm{x} \mathrm{g}$. Aliquots $(20 \mu \mathrm{l})$ of the supernatant were then assessed for the rate of apoptosis using the Cell Death Detection ELISA ${ }^{\text {PLUS }}$ kit (Roche Molecular Biochemicals). Quantitification of histone-associated DNA fragments were determined at $405 \mathrm{~nm}$ using a microplate ELISA reader (Varioskan LUX; Thermo Fisher Scientific).

Assessment of apoptosis. Annexin V-fluorescein isothiocyanate (FITC) and propidium iodide (PI) (Beyotime Institute of Biotechnology, Shanghai, China) staining were performed according to the manufacturer's instructions, and the cells were analyzed using flow cytometry (BD FACSCalibur; BD Biosciences, San Jose, CA, USA). Briefly, the cells were harvested by trypsinization and washed in PBS, and $1 \times 10^{5}$ cells were resuspended in $195 \mu \mathrm{l}$ binding buffer (Roche Molecular Biochemicals). The cell solution (195 $\mu \mathrm{l} ; 1 \times 10^{5}$ cells) were transferred to a $5 \mathrm{ml}$ culture tube and incubated with $5 \mu \mathrm{l}$ Annexin V-FITC and $10 \mu \mathrm{l}$ PI for $15 \mathrm{~min}$ at $25^{\circ} \mathrm{C}$ in the dark. Following incubation, $300 \mu \mathrm{l}$ binding buffer was added to each tube and the cells were analyzed using flow cytometry within $1 \mathrm{~h}$. The cells, which stained positive for Annexin V-FITC and negative for PI were considered to be undergoing early stage apoptosis. Cells that stained positive for Annexin V-FITC and PI were considered to be undergoing late stage apoptosis and those that stain negative for Annexin V-FITC and PI remained alive.

A terminal deoxynucleotidyltransferase-mediated dUTP nick-end labeling (TUNEL) assay was performed to identify apoptotic cells with fragmented DNA, which was performed using a in situ cell death detection kit, according to the manufacturer's instructions (Roche Molecular Biochemicals). Briefly, the hFOB1.19 cells $\left(5 \times 10^{3}\right)$ were plated onto coverslips and cultured overnight at $37^{\circ} \mathrm{C}$ and reached $80-90 \%$ confluency. The cells were then cultured in medium with different concentrations of puerarin $\left(10^{-8}-10^{-9} \mathrm{M}\right)$ at $37^{\circ} \mathrm{C}$ for $3 \mathrm{~h}$, and then treated with $10^{-5} \mathrm{M} \mathrm{DEX}$ at $37^{\circ} \mathrm{C}$ for $48 \mathrm{~h}$. Subsequently, the cells were fixed in immune stationary liquid (Beyotime Institute of Biotechnology, Suzhou, China) at $37^{\circ} \mathrm{C}$ for $60 \mathrm{~min}$ at room temperature and washed with immune washing liquid, containing 0.1\% Triton X-100 (contained in immune washing liquid; Beyotime Institute of Biotechnology, Suzhou, China), for 2 min on ice. For counterstaining, 4-Diamino-2-phenylindole (DAPI) was used, and the $\mathrm{TUNEL}^{+}$and $\mathrm{DAPI}^{+}$nuclei in the cells were counted manually. The cells were observed using an 
Table I. Primer sequences.

\begin{tabular}{lll}
\hline Name & Forward primers $\left(5^{\prime} \rightarrow 3^{\prime}\right)$ & Reverse primers $\left(5^{\prime} \rightarrow 3^{\prime}\right)$ \\
\hline Caspase-3 & TGTGAGGCGGTTGTAGAAGTT & GCTGCATCGACATCTGTACC \\
Bax & GTCCAATGTCCAGCCCATGA & ATCATGTTTGAGACCTTCAACA \\
Bcl-2 & GGTGAACTGGGGGAGGATTG & GGCAGGCATGTTGACTTCAC \\
-actin & ATCATGTTTGAGACCTTCAACA & CATCTCTTGCTCGAAGTCCA
\end{tabular}



Figure 1. Puerarin promotes hFOB1.19 cell proliferation. Cells proliferation was measured using an MTS detection kit. The cells were treated with $0 \mathrm{M}$ and $10^{-6}-10^{-10} \mathrm{M}$ puerarin for 3, 12 and $24 \mathrm{~h}$. Treatment with $10^{-8}$ and $10^{-9} \mathrm{M}$ puerarin significantly increased cell proliferation. Data are presented as the mean \pm standard deviation. ${ }^{*} \mathrm{P}<0.05$, vs control. OD, optical density.

Eclipse E600 fluorescence microscope (magnification, x100; Nikon, Tokyo, Japan), in which six fields were randomly selected. The percentage of positive cells was calculated as the apoptotic index (AI) using the following equation: $\mathrm{AI}=$ (number of positive cells / total number of cells) $\times 100 \%$, as previously described $(26,27)$.

Reverse transcription-quantitative polymerase chain reaction $(R T-q P C R)$. The total RNA was extracted from the cells using a MiniBEST Universal RNA Extraction kit (Takara Bio, Inc., Dalian, China). The concentration and purity of the total RNA were calculated with absorbance of 260 and $280 \mathrm{~nm}$. Single strand cDNA synthesis was performed using a PrimeScript RT Master Mix kit (Takara, Bio, Inc.). The qPCR was performed using SYBR Premix Ex Taq (Takara, Bio, Inc.) on an Mx3000P Real-Time PCR system (Applied Biosystems, Foster City, CA, USA) as follows: 50 cycles of $95^{\circ} \mathrm{C}$ for $10 \mathrm{sec}$ and $60^{\circ} \mathrm{C}$ for $30 \mathrm{sec}$. Each reaction contains $10 \mu \mathrm{l} \mathrm{SYBR}$ Green 1, $2 \mu \mathrm{l}$ primer, $1 \mu \mathrm{ldNTP}, 2 \mu \mathrm{l}$ Taq Polymerase, $5 \mu \mathrm{l} \mathrm{cDNA}$ and $30 \mu \mathrm{l}$ $\mathrm{ddH}_{2} \mathrm{O}$. All the reactions were repeated at least three times. The expression levels of the target genes, caspase-3, Bcl-2 and Bax, were calculated relative to housekeeping $\beta$-actin using Stratagene Mx3000P software (Applied Biosystems). The qPCR primers were designed using Primer 5.0 software, the sequences of which are listed in Table I.

Western blot analysis. Total protein was extracted from the cells using radioimmunoprecipitation assay lysis buffer and quanti- fied using a bicinchoninic acid protein assay (Beyotime Institute of Biotechnology, Suzhou, China). Equal quantities of protein were separated by sodium dodecyl sulfate-polyacrylamide gel electrophoresis (SDS-PAGE; 10\% gel) and transferred onto polyvinylidene difluoride membranes (EMD Millipore, Billerica, MA, USA). The membranes were then blocked with 5\% nonfat milk in PBS for $1 \mathrm{~h}$ at room temperature and incubated with Bcl-2 (rabbit mAb; 2870), Bax (rabbit mAb; 5023), cytochrome $c$ (rabbit mAb; 11940), Akt (rabbit mAb; 4691), phosphotylated (p)-Akt (Ser473; rabbit mAb; 4060), ERK1/2 (Rabbit Ab; 9102), p-ERK (rabbit mAb; 4370), p38 (rabbit mAb; 8690), p-p38 (rabbit mAb; 4511), JNK (rabbit Ab; 9252) and p-JNK (rabbit mAb; 4668) antibodies (1:1,000; Cell Signaling Technology, Inc., Beverly, MA, USA) overnight at $4^{\circ} \mathrm{C}$. Following extensive washing in washing liquid 3 times, the membranes were re-probed with horseradish peroxidase (HRP)-conjugated secondary antibodies (Cell Signaling Technology, Inc.). The blots were then washed, and the signal was visualized using an HRP chemiluminescent substrate reagent kit (Invitrogen Life Technologies, Carlsbad, CA, USA), according to the manufacturer's instructions. The band intensity was quantified using densitometric analysis with ImageJ 1.36 software (National Institutes of Health, Bethesda, MD, USA), with the absorbance ratio of each protein to the internal reference presented as the relative quantity of target protein.

Statistical analysis. SPSS version 11.5 for Windows was used for all statistical analyses. All data are expressed as the mean \pm standard deviation. Statistical analyses of the data were performed using one-way analysis of variance. $\mathrm{P}<0.05$ was considered to indicate a statistically significant difference. All experiments were repeated at least three times.

\section{Results}

Effects of puerarin on the viability of hFOB1.19 cells. To examine the effect of puerarin on the survival of hFOB1.19 cells, the present study performed an MTS assay using the puerarin- and vehicle (E2)-treated cells. The cells were treated with different concentrations of puerarin $\left(0,10^{-6}-10^{-10} \mathrm{M}\right)$ or E2 $\left(10^{-5} \mathrm{M}\right)$ for different durations ( 3,12 and $24 \mathrm{~h}$; Fig. 1$)$. Treatment with lower concentrations of puerarin $\left(10^{-8}-10^{-9} \mathrm{M}\right)$ increased cell growth and viability at all time-points $(\mathrm{P}<0.05)$, compared with the untreated control cells. Notably, the hFOB1.19 cells treated with puerarin at $10^{-8} \mathrm{M}$ for $3 \mathrm{~h}$ exhibited the highest proliferation rate. By contrast, treatment with a higher dose of puerarin $\left(10^{-6} \mathrm{M}\right)$ had no significant effects on cell viability, compared with E2-treated and untreated cells. Therefore, the cells were treated with puerarin at a concentration of $10^{-8} \mathrm{M}$ for a duration of $3 \mathrm{~h}$ in the subsequent experiments. 


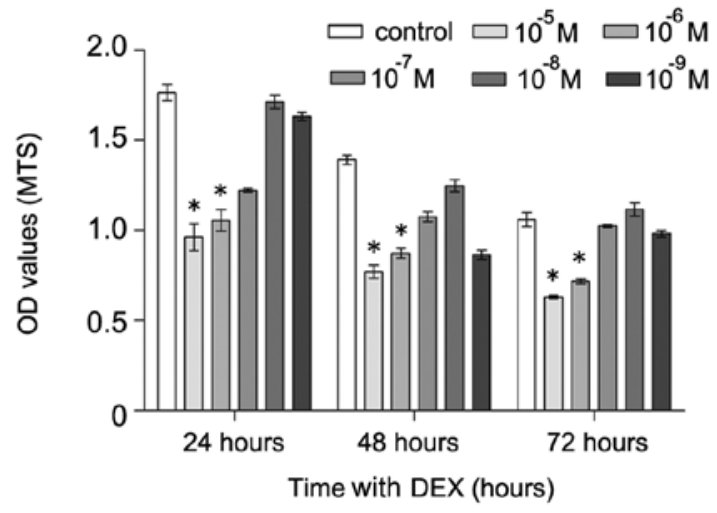

Figure 2. DEX inhibits hFOB1.19 cell proliferation. Cell proliferation was measured using an MTS detection kit following pretreatment of the cells with $0 \mathrm{M}$ and $10^{-5}-10^{-9} \mathrm{M}$ DEX for 24,48 and $72 \mathrm{~h}$. Treatment with $10^{-5}$ and $10^{-6} \mathrm{M}$ DEX significantly reduced cell proliferation. Data are presented as the mean \pm standard deviation. ${ }^{*} \mathrm{P}<0.05$, vs. control. DEX, dexamethasone; OD, optical density.

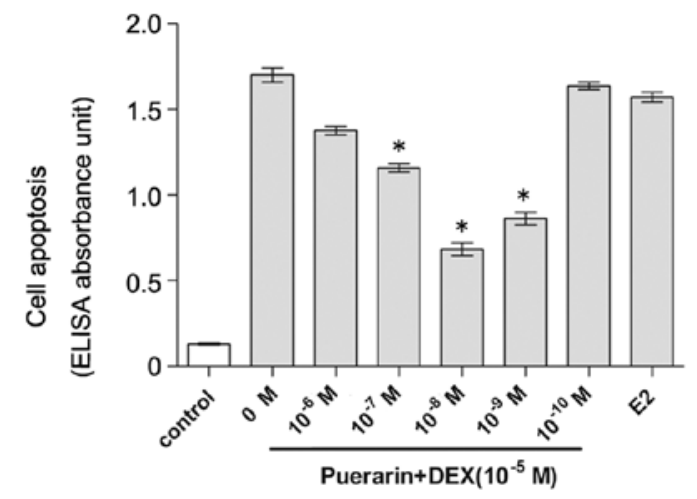

Figure 3. Purearin alleviates DEX-induced hFOB1.19 cell apoptosis. Cell apoptosis was measured using an enzyme-linked immunosorbent assay kit following pretreatment of the cells with $0 \mathrm{M}$ and $10^{-6}-10^{-10} \mathrm{M}$ puerarin for $3 \mathrm{~h}$. The cells were then incubated with DEX $\left(10^{-5} \mathrm{M}\right)$ for $48 \mathrm{~h}$. Treatment with $10^{-8}$ and $10^{-9} \mathrm{M}$ puerarin significantly reduced cell apoptosis. Data are presented as the mean \pm standard deviation. ${ }^{*} \mathrm{P}<0.05$, vs. DEX. DEX, dexamethasone.

DEX inhibits the proliferation of $h F O B 1.19$ cells. The hFOB1.19 cells were exposed to various concentrations $\left(0\right.$ and $\left.10^{-5}-10^{-9} \mathrm{M}\right)$ of DEX for 24,48 or $72 \mathrm{~h}$, and the cell viability was analyzed using an MTS assay. DEX had significant inhibitory effects on cell proliferation at concentration of $10^{-5}$ and $10^{-6} \mathrm{M}$ (Fig. 2). Treatment with DEX at $10^{-5} \mathrm{M}$ resulted in a 49,37 and $29 \%$ reduction of survival at 24,48 and $72 \mathrm{~h}$, respectively. Treatment with DEX at $10^{-6} \mathrm{M}$ resulted in a 35,30 and $25 \%$ reduction of survival at 24, 48 and $72 \mathrm{~h}$, respectively. Thus, the hFOB1.19 cells were treated with DEX at a concentration of $10^{-5} \mathrm{M}$ for a duration of $48 \mathrm{~h}$ in the subsequent experiments.

Puerarin suppresses DEX-induced apoptosis in hFOB1.19 cells, detected using ELISA and TUNEL. The present study used an ELISA to quantify DNA fragmentation, a hallmark of apoptosis (28). The hFOB1.19 cells were cultured in DEX $\left(10^{-5} \mathrm{M}\right)$-containing medium for $48 \mathrm{~h}$ in the presence of either $0-10^{-6} \mathrm{M}$ puerarin or $10^{-5} \mathrm{M}$ E2. The hFOB1.19 cells in $10 \%$ FBS medium exhibited basal levels of apoptosis
$\mathbf{A}$

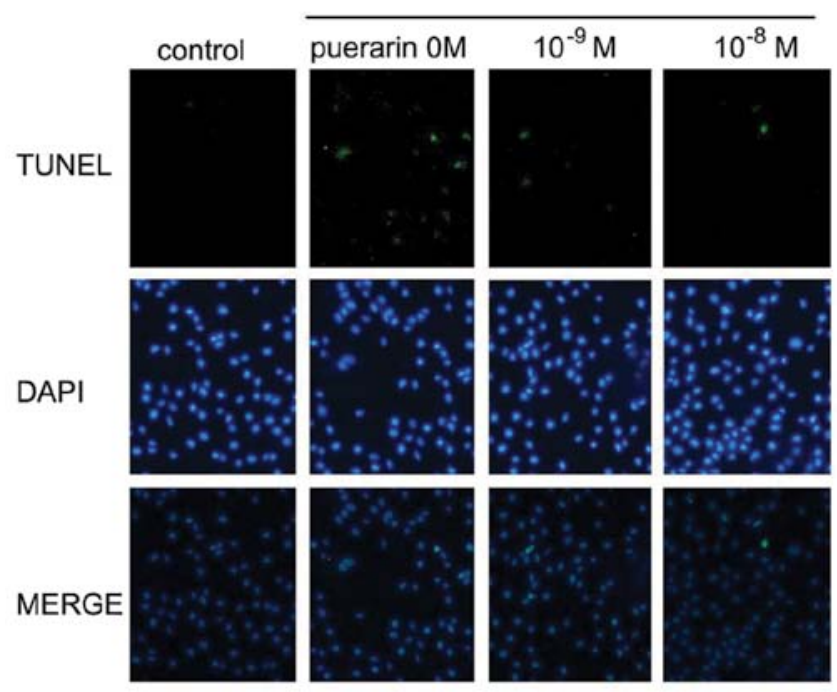

B

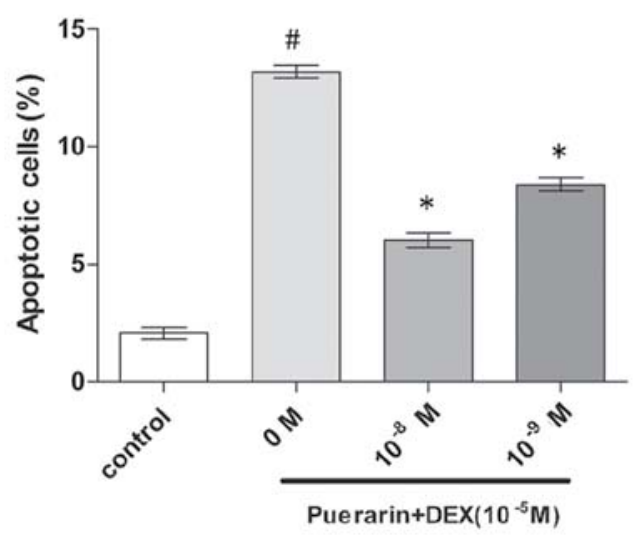

Figure 4. Effects of puerarin on apoptosis in hFOB1.19 cells, determined using a TUNEL assay. (A) Cells were pretreated with puerarin $\left(0\right.$ or $\left.10^{-8}-10^{-9} \mathrm{M}\right)$ for $3 \mathrm{~h}$, and were then incubated with DEX $\left(10^{-5} \mathrm{M}\right)$ for $48 \mathrm{~h}$. The number of apoptotic cells were determined using TUNEL staining and observed under a fluorescence microscope; original magnification, x100. (B) Percentages of positive cells were calculated in six randomly selected fields as the apoptotic index using the following equation: $\mathrm{AI}=$ (number of positive cells $/$ total number of cells) x $100 \%$. Arrows indicate apoptotic cells. Data are presented as the mean \pm standard deviation $(n=3)$. $P<0.05$, vs. DEX; ${ }^{*} \mathrm{P}<0.05$, vs. control. DEX, dexamethasone; TUNEL, terminal deoxynucleotidyltransferase-mediated dUTP nick-end labeling; DAPI, 4-Diamino-2-phenylindole.

$(0.13 \pm 0.01$ ELISA absorbance units), while DEX treatment markedly increased the levels of apoptosis, compared with the control (1.71 \pm 0.01 ELISA absorbance units; Fig. 3). However, following exposure to puerarin the numbers of apoptotic cells in the cells treated with puerarin at concentrations of $10^{-10} \mathrm{M}$ $(1.64 \pm 0.03), 10^{-9} \mathrm{M}(0.83 \pm 0.05), 10^{-8} \mathrm{M}(0.64 \pm 0.05), 10^{-7} \mathrm{M}$ $(1.16 \pm 0.05)$ and $10^{-6} \mathrm{M}(1.33 \pm 0.04$ ELISA absorbance units) were lower, compared with those in the DEX-treated group and positive control group exposed to $10^{-5} \mathrm{M}$ E2 $(\mathrm{P}<0.05)$. The maximal anti-apoptotic effect of puerarin was observed at a concentration of $10^{-8} \mathrm{M}$, and no anti-apoptotic effect was observed at a concentration of $10^{-10} \mathrm{M}$.

A TUNEL assay was also performed in the present study, which also indicated that $10^{-8} \mathrm{M}$ puerarin significantly decrease 
A

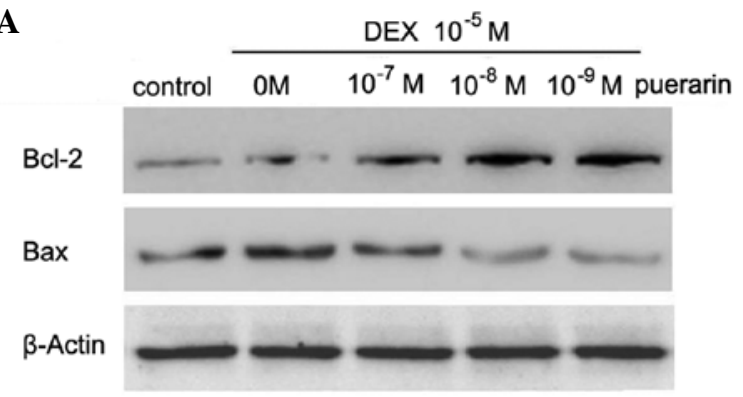

B

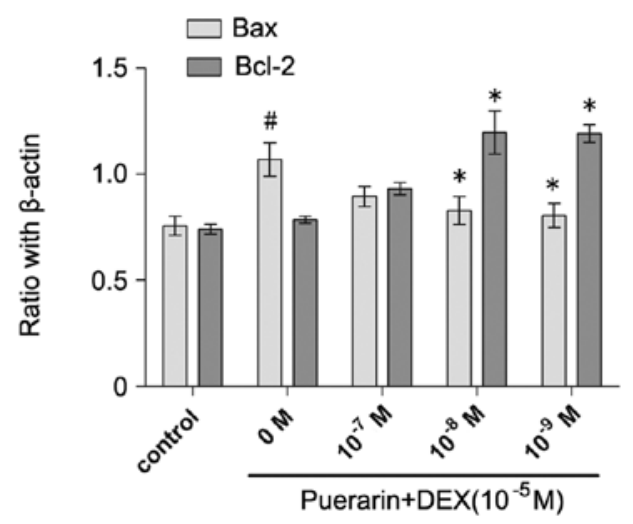

Figure 5. Effects of puerarin on DEX-induced changes in of Bcl-2 and Bax. (A) Cells were treated with puerarin $\left(0,10^{-7}, 10^{-8}\right.$ and $\left.10^{-9} \mathrm{M}\right)$ for $3 \mathrm{~h}$ prior to incubation with DEX $\left(10^{-5} \mathrm{M}\right)$ for $48 \mathrm{~h}$. Western blot analyses for Bcl-2, Bax and $\beta$-actin was performed. (B) Relative expression levels of Bcl-2 and Bax compared with $\beta$-actin. Data are presented as the mean \pm standard deviation $(\mathrm{n}=3) .{ }^{*} \mathrm{P}<0.05$, vs. DEX; ${ }^{\mathrm{P}} \mathrm{P}<0.05$, vs. control. DEX, dexamethasone; Bcl-2, B-cell lymphoma-2; Bax, B-cell-associated X protein.

DEX-induced apoptosis in the hOB1.19 cells. The hFOB1.19 cells were treated with $\operatorname{DEX}\left(10^{-5} \mathrm{M}\right)$ in the absence or presence of $10^{-8}$ and $10^{-9} \mathrm{M}$ puerarin. The percentage of apoptotic cells exhibiting green condensed or fragmented nuclei, identified by TUNEL staining, was significantly lower in the cells treated with DEX in the presence of puerarin (Fig. 4), compared with the cells in the control group. The data from the above two assays indicated that puerarin protected the hFOB1.19 cells against DEX-induced apoptosis.

Effect of puerarin on the expression of apoptosis-associated protein in hFOB1.19 cells. To clarify the possible mechanisms by which puerarin attenuated DEX-induced apoptosis, western blot analysis and RT-qPCR were performed to examine a panel of apoptosis-associated proteins, including Bcl-2, Bax, caspase-3 and cytochrome $c$. The hFOB1.19 cells were incubated with DEX $10^{-5} \mathrm{M}$ in combination with different concentrations of puerarin $\left(0,10^{-9}, 10^{-8}\right.$ and $\left.10^{-7} \mathrm{M}\right)$ for $48 \mathrm{~h}$, following which the protein and mRNA levels were examined.

The results demonstrated that puerarin increased the protein expression of $\mathrm{Bcl}-2$, and decreased the protein expression of $\mathrm{Bax}$ in the hFOB1.19 cells in a dose-dependent manner (all $\mathrm{P}<0.05$; Fig. 5). As the concentration of puerarin increased between $10^{-7}$ and $10^{-9} \mathrm{M}$, the protein expression of Bax decreased gradually, and $10^{-9} \mathrm{M}$ puerarin almost eradicated the DEX-induced expression of $\mathrm{Bax}(\mathrm{P}<0.05)$, compared with the cells treated

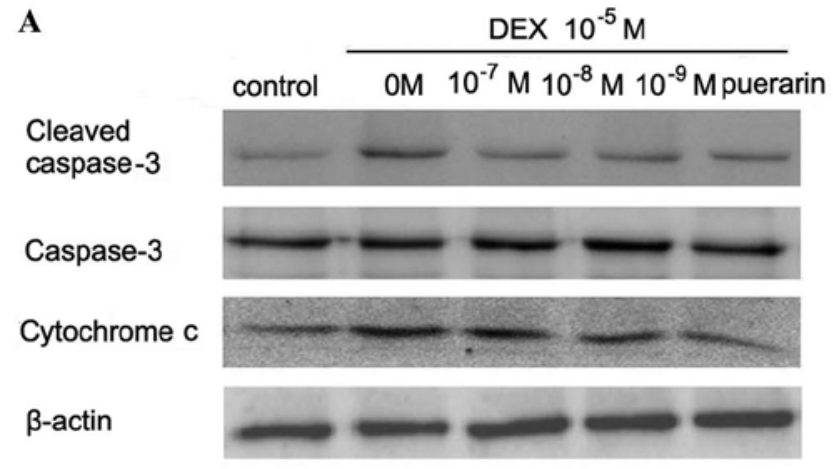

B

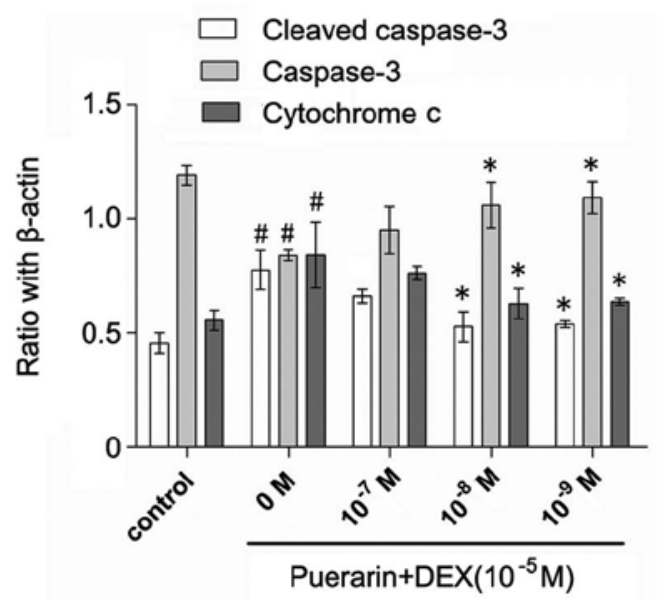

Figure 6. Effects of puerarin on the expression levels of caspase-3 and cytochrome $c$ in the hFOB1.19 cells. (A) Cells were treated with puerarin $\left(0,10^{-7}, 10^{-8}\right.$ and $\left.10^{-9} \mathrm{M}\right)$ for $3 \mathrm{~h}$ prior to incubation with $\operatorname{DEX}\left(10^{-5} \mathrm{M}\right)$ for $48 \mathrm{~h}$. Western blot analyses were performed to detect the expression levels of caspase-3, cleaved caspase-3, cytochrome $c$, and $\beta$-actin. (B) Relative expression levels of cleaved caspase- 3 and cytochrome $c$ were compared with the expression levels of total caspase- 3 and $\beta$-actin. Data are presented as the mean \pm standard deviation $(\mathrm{n}=3) .{ }^{*} \mathrm{P}<0.05$, vs. DEX; ${ }^{\#} \mathrm{P}<0.05$, vs. control. DEX, dexamethasone.

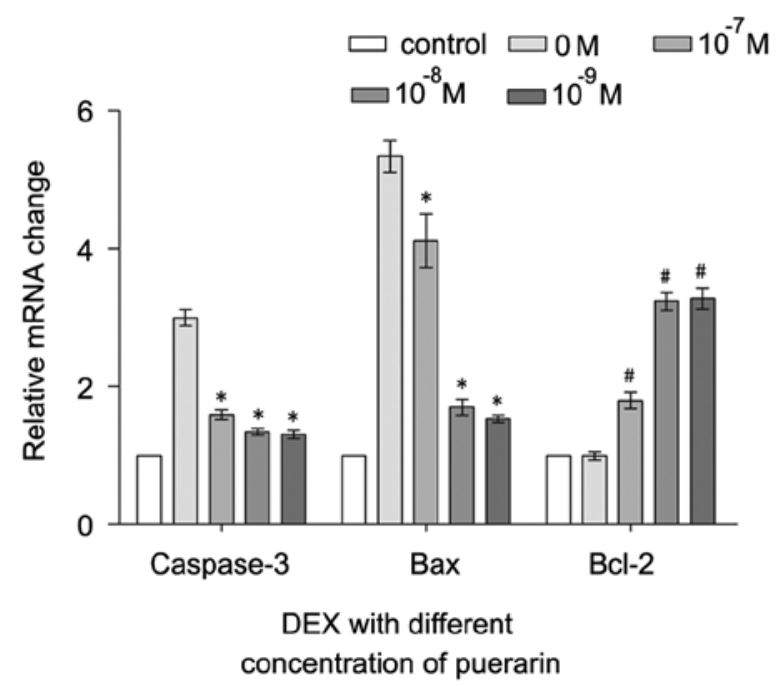

Figure 7. RT-qPCR analysis of caspase-3, Bax and Bcl-2. The cells were treated with puerarin $\left(0,10-7,10^{-8}\right.$ and $\left.10^{-9} \mathrm{M}\right)$ for $3 \mathrm{~h}$ prior to incubation with DEX $\left(10^{-5} \mathrm{M}\right)$ for $48 \mathrm{~h}$. RT-qPCR was performed to analyzed expression levels of caspase-3, Bax and Bcl-2. Data are presented as the mean \pm standard deviation. ${ }^{*} \mathrm{P}<0.05$, vs. DEX; ${ }^{\text {}} \mathrm{P}<0.05$, vs. control. RT-qPCR, reverse transcription-quantitative polymerase chain reaction; DEX, dexamethasone; Bcl-2, B-cell lymphoma-2; Bax, B-cell-associated X protein. 




B

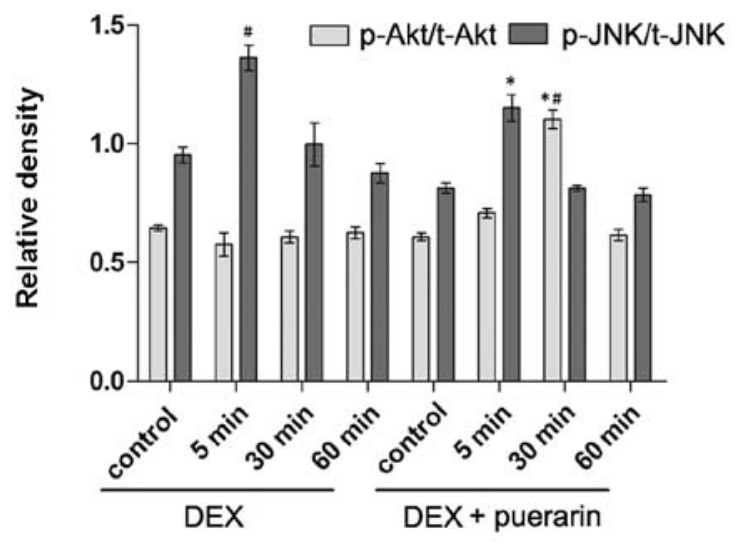

C

DEX $10^{-5} \mathrm{M} \quad$ DEX $10^{-5} \mathrm{M}+$ puerarin $10^{-8} \mathrm{M}$

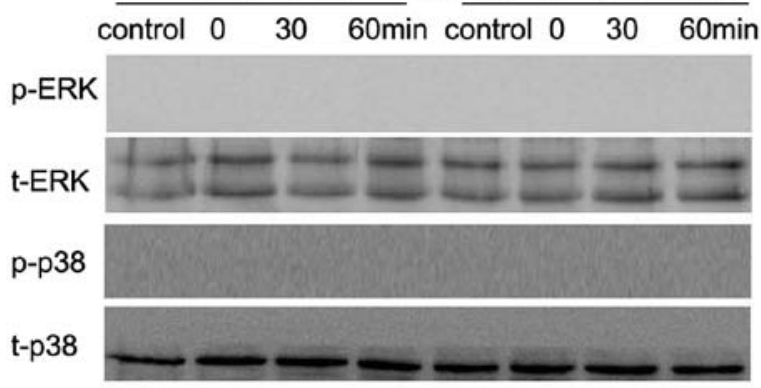

Figure 8. Effects of puerarin on the phosphorylation of JNK and Akt in hFOB1.19 cells. (A and C) Cells were treated with DEX $\left(10^{-5} \mathrm{M}\right)$ alone or in combination with puerarin $\left(10^{-8} \mathrm{M}\right)$ for 5, 30 and $60 \mathrm{~min}$. Western blot analysis was performed to detect the expression levels of p-JNK, JNK, p-Akt, Akt, p-ERK and ERK. (B) Relative expression levels of p-Akt and p-JNK compared with t-Akt and t-JNK were analyzed, respectively. Data are presented as the mean \pm standard deviation $(n=3)$. ${ }^{*} \mathrm{P}<0.05$, vs. DEX; ${ }^{\text {"}} \mathrm{P}<0.05$, vs. control. JNK, c-Jun $\mathrm{N}$-terminal kinase; ERK, extracellular signal-regulated kinase; p-, phosphorylated; t-, total.

with DEX only (Fig. 5). In addition, puerarin at concentrations of $10^{-8}$ and $10^{-9} \mathrm{M}$ increased the protein expression of $\mathrm{Bcl}-2$, and the maximal effect was observed at $10^{-8} \mathrm{M}(\mathrm{P}<0.05$, compared with DEX alone; Fig. 5). In accordance, the results of the qPCR revealed similar results to the western blot analyses.

Cytochrome $c$ was released into the cytoplasm in the DEX-treated cells, whereas pretreatment with puerarin at concentration between $10^{-7}$ and $10^{-9} \mathrm{M}$ attenuated the DEX-induced release of cytochrome $c$ (Fig. 6). Additionally, puerarin pretreatment significantly decreases the protein expression of cleaved caspase-3. Consistent with the results of cellular caspase activity, the results of the qPCR revealed that puerarin pretreatment significantly decreased the mRNA levels of caspase-3 (Fig. 7).

Puerarin inhibits the JNK pathway and activates the PI3K/Akt signaling pathway in hFOB1.19 cells. Puerarin exhibited no effects on p38 or extracellular signal-regulated kinase (ERK) phosphorylation, however, it increased the levels of p-Akt, and inhibited the levels of p-JNK (Fig. 8). The hFOB1.19 cells were treated with $\operatorname{DEX}\left(10^{-5} \mathrm{M}\right)$ alone or in combination with puerarin $\left(10^{-8} \mathrm{M}\right)$ for 5,30 or $60 \mathrm{~min}$, respectively. The results revealed that DEX significantly increased the expression of p-JNK, with a maximal effect after $5 \mathrm{~min}$, however, no effect on the expression of $\mathrm{p}$-Akt was observed. Notably, the combined treatment with puerarin ameliorated the DEX-induced activation of the JNK pathway, which became apparent at $30 \mathrm{~min}$ and peaked $60 \mathrm{~min}$ after puerarin treatment. In addition, puerarin induced the phosphorylation of Akt, which peaked after $30 \mathrm{~min}$.

Effect of LY294002and SP600125 on puerarin-induced changes in the expression levels of p-Akt, p-JNK in hFOB1.19 cells. In order to clarify whether the PI3K/Akt inhibitor, LY294002, or JNK inhibitor, SP600125, had any effect on puerarin-induced phosphoralation changes of Akt and JNK, the present study incubated the cells with DEX, puerarin, LY294002 and SP600125, alone or in combination, for $30 \mathrm{~min}$ (p-Akt) or 5 min (p-JNK) (Fig. 9). The results demonstrated that puerarin combined with DEX increased the phosphorylation of Akt, compared with DEX alone, however, LY294002 partially abrogated this effect. The JNK inhibitor, SP600125, ameliorated DEX-induced phosphorylation of JNK (Fig. 10). In addition, puerarin combined with SP600125 almost eliminated the DEX-induced expression of p-JNK, indicating puerarin as a potent JNK suppressor, which had synergistic effect with SP600125.

JNK and PI3K/Akt signaling pathways mediate the inhibitory effects of puerarin on apoptosis in hFOB1.19 cells. As puerarin treatment was observed to inhibit the JNK signaling pathway and activate the PI3K/Akt signaling pathway in the hFOB1.19 cells, and it is well known that these two pathways are closely associated with the regulation of apoptosis, the present study further examined whether these two pathways are involved in the anti-apoptotic activity of puerarin. Cell apoptosis was determined using Annexin V-FITC/PI flow cytometric analysis (Fig. 10). According to the results, DEX treatment significantly induced apoptosis, while combined treatment with puerarin significantly attenuated this effect. In addition, inhibition of the Akt pathway by LY294002 caused a marginal increase in DEX-induced apoptosis, and LY294002 partly eliminated the protective effect of puerarin on DEX-induced apoptosis, indicating that puerarin exhibited anti-apoptotic properties, partly dependent on the Akt signaling pathway. In line with the above results, puerarin combined with SP600125 suppressed DEX-apoptosis to a lesser extent, compared with SP600125 alone, suggesting that puerarin enhanced the anti-apoptotic effect of the JNK inhibitor, and that combination treatment may be a potentially therapeutic option for GC-induced osteoporosis. 
A

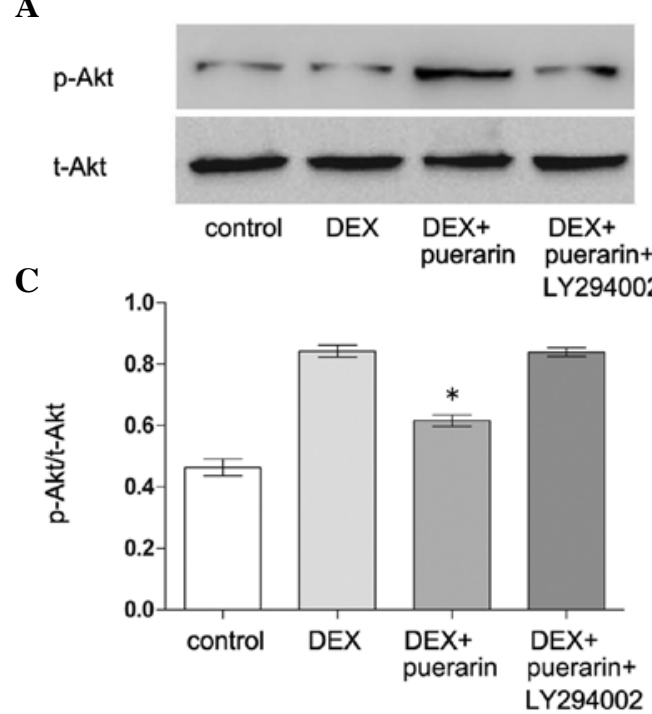

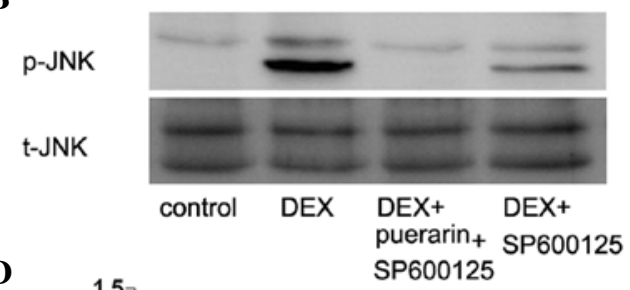

D

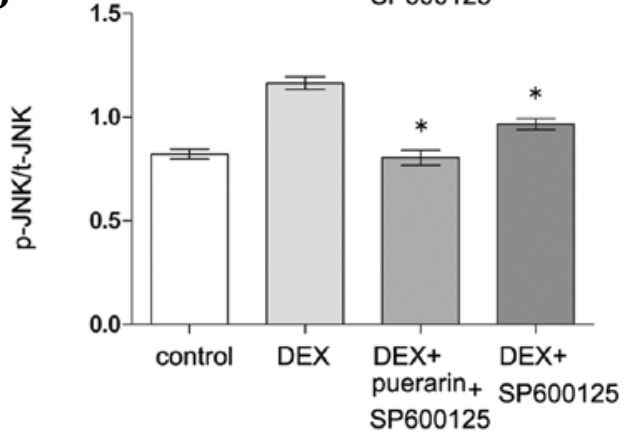

Figure 9. Effects of LY294002 and SP600125 on the expression levels of p-Akt and p-JNK in hFOB1.19 cells. (A) Cells were treated with DEX (10 $\left.{ }^{-5} \mathrm{M}\right)$, DEX $\left(10^{-5} \mathrm{M}\right)+\mathrm{LY} 294002\left(10^{-5} \mathrm{M}\right)$, or DEX $\left(10^{-5} \mathrm{M}\right)+$ puerarin $\left(10^{-8} \mathrm{M}\right)+\mathrm{LY} 294002\left(10^{-5} \mathrm{M}\right)$. Expression levels of p-Akt and t-Akt were detected using western blot analysis. (B) Cells were treated with DEX $\left(10^{-5} \mathrm{M}\right)$, DEX $\left(10^{-5} \mathrm{M}\right)+\operatorname{SP} 600125\left(10^{-5} \mathrm{M}\right)$, or DEX $\left(10^{-5} \mathrm{M}\right)+$ puerarin $\left(10^{-8} \mathrm{M}\right)+\mathrm{SP} 600125\left(10^{-5} \mathrm{M}\right)$. Expression levels of p-JNK and t-JNK were detected using western blot analysis. Data are presented as the mean \pm standard deviation. " $\mathrm{P}<0.05$, vs. DEX. JNK, c-Jun N-terminal kinase; p-, phosphorylated; t-, total.

$\mathbf{A}$

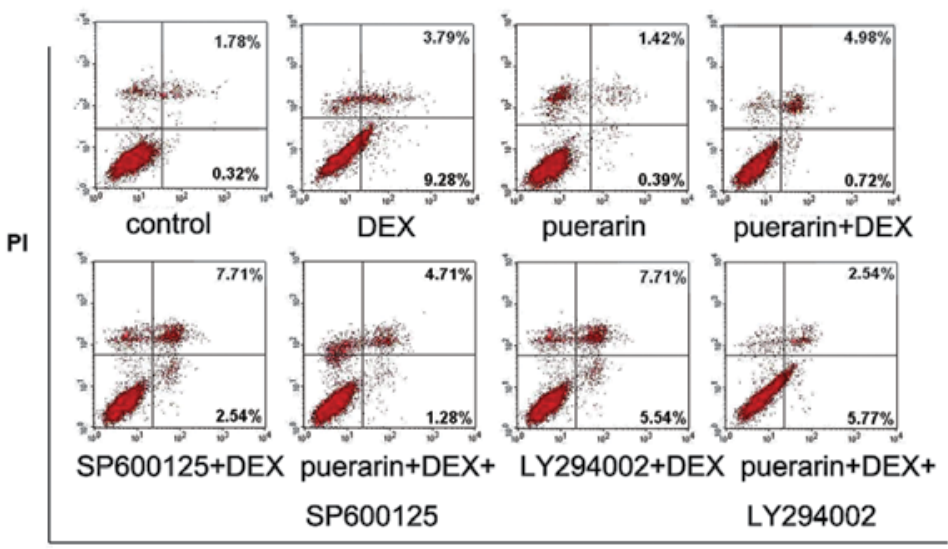

FITC-Annexin V
B

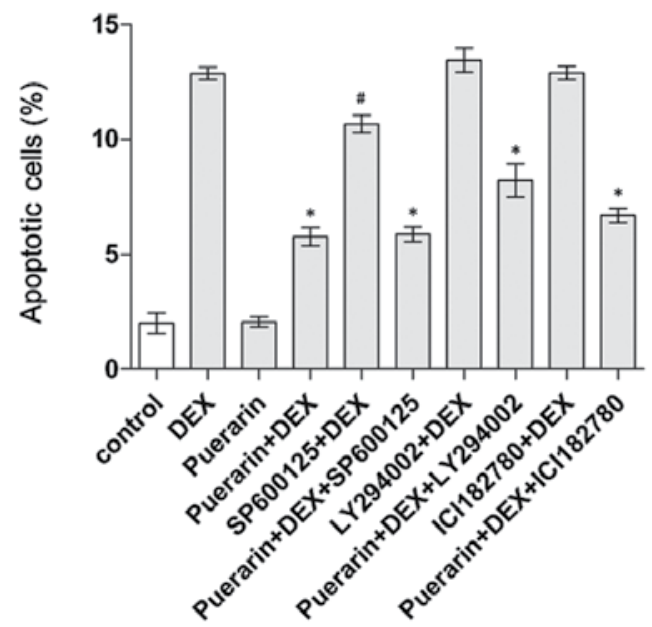

Figure 10. Effects of JNK and Akt on the apoptosis of hFOB1.19 cells. (A) Cells were treated with puerarin $\left(10^{-8} \mathrm{M}\right)$, SP600125 (10 $\left.0^{-5} \mathrm{M}\right)$ or LY294002 (10 $\left.0^{-5} \mathrm{M}\right)$ for $3 \mathrm{~h}$ prior to incubation with DEX $\left(10^{-5} \mathrm{M}\right)$ for $48 \mathrm{~h}$. Flow cytometric analysis was performed following Annexin V-FITC/PI double staining. The upper left quadrant represents dead cells or debris, the upper right quadrant represents the late stage of apoptotic cells, the lower right quadrant represents the early stage of apoptotic cells and the lower left quadrant represented normal cells. (B) Levels of apoptosis of the hFOB1.19 cells were analyzed. Data are presented as the mean \pm standard deviation. ${ }^{\text {P }}<0.01$, vs. DEX; ${ }^{\text {P }}<0.05$, vs. control. DEX, dexamethasone; FITC, fluorescein isothiocyanate; PI, propidium iodide.

\section{Discussion}

GCs are widely used for their unsurpassed anti-inflammatory and immunomodulatory effects, however, the clinical applications are limited by substantial adverse outcomes, including osteoporosis (29). GIOP is the most common cause of secondary osteoporosis (30) and the pro-apoptotic effect of high-dose GCs on osteoblasts has been identified as a major cause for GIOP $(4,31,32)$. However, the underlying molecular mechanisms underlying this action remain to be elucidated, which has impeded the prevention and cure of this side effect.
The identification of naturally occurring phytochemicals, which can antagonize osteoporosis has received increased attention. Studies have demonstrated that dehydrocostus lactone, puerarin and chemical constituents of the fruits of Prunus mume can reverse GC-induced apoptosis in osteoblasts by regulating of various signaling pathways $(10,33,34)$. Puerarin, the major isoflavonoid in the traditional Chinese herb pueraria lobata, is unique in that it contains a C-C conjugated glucose at position 8 of the isoflavonoid structure (35), and has been widely used in traditional Chinese medicine for the treatment of various diseases $(36,37)$. Puerarin is reported 
to significantly facilitate the survival rate of osteoblasts, and regulate osteoblast proliferation and differentiation. In vivo studies have demonstrated that osteoblast implants in the puerarin-treated rats have a higher rate of bone formation $(38,39)$. In addition, puerarin in the diet has been observed to effectively prevent osteoporosis in ovariectomized rats by increasing bone mineral density and bone mineral content $(35,40)$. Furthermore, bone defects, created in rabbit parietal bone grafted using a mixture of puerarin and collagen, formed $>5$-fold more new bone, compared with defects grafted with collagen alone (41). In addition, various studies have reported that puerarin stimulates the proliferation and differentiation of osteoblasts and protects osteoblasts against cell death in vitro $(10,17,35,42)$. In the present study, puerarin attenuated DEX-induced apoptosis in hFOB1.19 cells through activation of the PI3K/Akt signaling pathway and inhibition of the JNK signaling pathway. In addition, puerarin upregulated Bcl-2, downregulated Bax, and inhibited the cleavage of caspase-3 and release of cytochrome $c$. These findings indicated that puerarin exhibited anti-apoptotic properties through the mitochondrial apoptotic pathway.

Mitochondria have been reported to be key in the regulation of apoptosis. The activation of Bax triggers the release of cytochrome $c$ from the intermembrane space into the cytoplasm, which finally activates caspase enzymes that mediate the entrance in the execution phase of the apoptotic program (43). Anti-apoptotic Bcl-2-associated proteins suppress the activities of Bax and prevent the release of cytochrome $c$ (44). Previous studies have demonstrated that puerarin suppresses apoptosis through regulation of the $\mathrm{Bcl}-2$ family proteins in a variety of cells, including vascular endothelial cells, osteobastic cells, vascular smooth muscle cells and human neurons (10,45-47). In the present study, western blot analysis and RT-qPCR were used to examine the effect of puerarin on major members of the Bcl-2 family, including anti-apoptotic Bcl-2 and pro-apoptotic Bax (48). The results demonstrated that DEX repressed the expression of Bcl-2 and promoted the release of Bax. Puerarin attenuated these changes, indicating that puerarin exerted its activities by modulating Bcl-2 and Bax in hFOB1.19 cells.

Caspase- 3 is one of the major activated cysteine proteases in the caspase family and is pivotal in apoptosis. Caspase-3 was considered to be a central mediator of apoptosis, it cleaves a number of substrates and activates endonucleases, including the activation of caspase-activated DNase, which is responsible for internucleosomal DNA fragmentation, a hallmark of apoptosis (49-51). In addition to activation of the caspase family, the release of cytochrome $c$ is regarded as a key regulatory event in apoptosis under certain conditions and is released from mitochondria into the cytosol, which results in caspase activation and cell apoptosis (52). It is reported that osteoblasts undergoing apoptosis in response to GCs exhibit typical features, including the activation of caspase- 3 and release of cytochrome $c(53,54)$. In the present study, DEX induced the cleavage of caspase- 3 and the release of cytochrome $c$, whereas treatment with puerarin suppressed the cleavage of caspase-3 and release of cytochrome $c$, suggesting that puerarin inhibited DEX-induced apoptosis in a mitochondria-dependent manner.

To further investigate the detailed mechanisms and signaling pathways involved in the anti-apoptotic activity of puerarin, the present study investigated the responses of the
PI3K/Akt and MAPK serine/threonine kinase signaling pathways to puerarin treatment in the hFOB1.19 cells.

MAPK pathways, including the ERK, JNK and p38 pathways, are activated by several stimuli, and one of their major functions is to connect cell surface receptors to transcription factors in the nucleus, which consequently triggers long-term cellular responses (54). ERK1/2 is generally associated with proliferation and growth. By contrast, the JNK and p38 pathways are pivotal in mediating apoptosis by modulating the transcription of apoptosis-associated genes, and is involved in the progression of apoptosis in osteoblasts (17-20,27,55-57). The present study provided the first evidence, to the best of our knowledge, that the JNK-MAPK pathways mediated the inhibitory effects of puerarin on DEX-induced apoptosis in the hFOB1.19 cells.

Among the pathways linked to apoptosis resistance, the PI3K/Akt pathway stands out as the convergent point for a variety of stimuli generated at the cell surface $(58,59)$. The PI3K/Akt pathway, which can be activated by growth factors and certain extracellular signals, regulates cell proliferation, differentiation and survival (60). It has been reported that the $\mathrm{PI} 3 \mathrm{~K} / \mathrm{Akt}$ pathway is involved in the protection of osteoblasts from apoptosis $(21,22,61)$. The present study investigated whether puerarin affected the activation of PI3K/Akt, and found that puerarin increased the phosphorylation of Akt. In addition, the involvement of the PI3K/Akt signaling pathway in the anti-apoptotic activity of puerarin was examined. The results revealed that PI3K inhibitor, LY294002, partially abrogated the anti-apoptotic effects of puerarin, confirming that the PI3K/Akt pathway is critical in the anti-apoptotic effect of puerarin against DEX-induced apoptosis in hFOB1.19 cells.

In conclusion, the present study demonstrated that puerarin prevented the DEX-induced apoptosis of hFOB1.19 cells via inhibition of the JNK pathway and activation of the PI3K/Akt signaling pathway in hFOB1.19 cells, which was dependent on the mitochondrial apoptotic pathway. These results indicated puerarin as a promising target in the treatment of GC-induced osteoporosis.

\section{Acknowledgements}

This study was supported by grants from the National Natural Science Foundation of China (grant. nos. 81370981 and 81200714).

\section{References}

1. Canalis E: Clinical review 83: Mechanisms of glucocorticoid action in bone: implications to glucocorticoid-induced osteoporosis. J Clin Endocrinol Metab 81: 3441-3447, 1996.

2. Manelli F and Giustina A: Glucocorticoid-induced osteoporosis. Trends Endocrinol Metab 11: 79-85, 2000.

3. Tamura Y, Okinaga $\mathrm{H}$ and Takami H: Glucocorticoid-induced osteoporosis. Biomed Pharmacother 58: 500-504, 2004.

4. Weinstein RS, Jilka RL, Parfitt AM and Manolagas SC: Inhibition of osteoblastogenesis and promotion of apoptosis of osteoblasts and osteocytes by glucocorticoids. Potential mechanisms of their deleterious effects on bone. J Clin Invest 102: 274-282, 1998.

5. Wu L, Qiao H, Li Y and Li L: Protective roles of puerarin and Danshensu on acute ischemic myocardial injury in rats. Phytomedicine 14: 652-658, 2007. 
6. Guerra MC, Speroni E, Broccoli M, Cangini M, Pasini P, Minghett A, Crespi-Perellino N, Mirasoli M, Cantelli-Forti G and Paolini M: Comparison between chinese medical herb Pueraria lobata crude extract and its main isoflavone puerarin antioxidant properties and effects on rat liver CYP-catalysed drug metabolism. Life Sci 67: 2997-3006, 2000.

7. Dong LP and Wang TY: Effects of puerarin against glutamate excitotoxicity on cultured mouse cerebral cortical neurons. Zhongguo Yao Li Xue Bao 19: 339-342, 1998.

8. Huang F, Liu K, Du H, Kou J and Liu B: Puerarin attenuates endothelial insulin resistance through inhibition of inflammatory response in an IKK $\beta /$ IRS-1-dependent manner. Biochimie 94: 1143-1150, 2012.

9. Liu CM, Ma JQ and Sun YZ: Puerarin protects the rat liver against oxidative stress-mediated DNA damage and apoptosis induced by lead. Exp Toxicol Pathol 64: 575-582, 2012.

10. Wang Y, Wang WL, Xie WL, Li LZ, Sun J, Sun WJ and Gong HY: Puerarin stimulates proliferation and differentiation and protects against cell death in human osteoblastic MG-63 cells via ER-dependent MEK/ERK and PI3K/Akt activation. Phytomedicine 20: 787-796, 2013.

11. Liu CM, Ma JQ and Sun YZ: Puerarin protects rat kidney from lead-induced apoptosis by modulating the PI3K/Akt/eNOS pathway. Toxicol Appl Pharmacol 258: 330-342, 2012.

12. Li J, Wang G, Liu J, Zhou L, Dong M, Wang R, Li X, Li X, Lin $\mathrm{C}$ and Niu Y: Puerarin attenuates amyloid-beta-induced cognitive impairment through suppression of apoptosis in rat hippocampus in vivo. Eur J Pharmacol 649: 195-201, 2010.

13. Abud HE: Shaping developing tissues by apoptosis. Cell Death Differ 11: 797-799, 2004

14. Elmore S: Apoptosis: A review of programmed cell death Toxicol Pathol 35: 495-516, 2007.

15. Adams JM and Cory S: The Bcl-2 protein family: Arbiters of cell survival. Science 281: 1322-1326, 1998.

16. Zhang H, Liu Y, Lao M, Ma Z and Yi X: Puerarin protects Alzheimer's disease neuronal cybrids from oxidant-stress induced apoptosis by inhibiting pro-death signaling pathways Exp Gerontol 46: 30-37, 2011

17. Liu LJ, Liu LQ, Bo T, Li SJ, Zhu Z, Cui RR and Mao DA Puerarin suppress apoptosis of human osteoblasts via ERK signaling pathway. Int J Endocrinol 2013: 786574, 2013.

18. Wang G, Zhou L, Zhang Y, Dong M, Li X, Liu J and Niu Y: Implication of the c-Jun-NH2-terminal kinase pathway in the neuroprotective effect of puerarin against 1-methyl-4-phenylpyridinium (MPP+)-induced apoptosis in PC-12 cells. Neurosci Lett 487: 88-93, 2011.

19. Guo C, Yuan L, Wang JG, Wang F, Yang XK, Zhang FH, Song JL, Ma XY, Cheng Q and Song GH: Lipopolysaccharide (LPS) induces the apoptosis and inhibits osteoblast differentiation through JNK pathway in MC3T3-E1 cells. Inflammation 37: 621-631, 2014

20. Tang SY, Xie H, Yuan LQ, Luo XH, Huang J, Cui RR, Zhou HD, Wu XP and Liao EY: Apelin stimulates proliferation and suppresses apoptosis of mouse osteoblastic cell line MC3T3-E1 via JNK and PI3-K/Akt signaling pathways. Peptides 28 708-718, 2007

21. Liang QH, Liu Y, Wu SS, Cui RR, Yuan LQ and Liao EY: Ghrelin inhibits the apoptosis of MC3T3-E1 cells through ERK and AKT signaling pathway. Toxicol Appl Pharmacol 272: 591-597, 2013.

22. Liang D, Xiang L, Yang M, Zhang X, Guo B, Chen Y, Yang L and Cao J: ZnT7 can protect MC3T3-E1 cells from oxidative stress-induced apoptosis via PI3K/Akt and MAPK/ERK signaling pathways. Cell Signal 25: 1126-1135, 2013.

23. Feng R, Feng L, Yuan Z, Wang D, Wang F, Tan B, Han S, Li T, Li D and Han Y: Icariin protects against glucocorticoid-induced osteoporosis in vitro and prevents glucocorticoid-induced osteocyte apoptosis in vivo. Cell Biochem Biophys 67: 189-197, 2013.

24. Guo D, Li Q, Lv Q, Wei Q, Cao S and Gu J: MiR-27a targets sFRP1 in hFOB cells to regulate proliferation, apoptosis and differentiation. PLoS One 9: e91354, 2014.

25. Cui RR, Mao DA, Yi L, et al: Apelin suppresses apoptosis of human vascular smooth muscle cells via APJ/PI3-K/Akt signaling pathways. Amino Acids 39: 1193-1200, 2010.

26. Kitamura T, Itoh M, Noda T, Matsuura M and Wakabayashi K: Combined effects of cyclooxygenase- 1 and cyclooxygenase-2 selective inhibitors on intestinal tumorigenesis in adenomatous polyposis coli gene knockout mice. Int J Cancer 109: 576-580, 2004.

27. Zhu X, Jiang Y, Shan PF, et al: Vaspin attenuates the apoptosis of human osteoblasts through ERK signaling pathway. Amino Acids 44: 961-968, 2013.
28. Habata Y, Fujii R, Hosoya M, et al: Apelin, the natural ligand of the orphan receptor APJ, is abundantly secreted in the colostrum. Biochim Biophys Acta 1452: 25-35, 1999.

29. Seibel MJ, Cooper MS and Zhou H: Glucocorticoid-induced osteoporosis: Mechanisms, management, and future perspectives. Lancet Diabetes Endocrinol 1: 59-70, 2013.

30. van Brussel MS, Bultink IE and Lems WF: Prevention of glucocorticoid-induced osteoporosis. Expert Opin Pharmacother 10: 997-1005, 2009.

31. Beavan S, Horner A, Bord S, Ireland D and Compston J: Colocalization of glucocorticoid and mineralocorticoid receptors in human bone. J Bone Miner Res 16: 1496-1504, 2001.

32. Gohel A, McCarthy MB and Gronowicz G: Estrogen prevents glucocorticoid-induced apoptosis in osteoblasts in vivo and in vitro. Endocrinology 140: 5339-5347, 1999.

33. Choi EM, Kim GH and Lee YS: Protective effects of dehydrocostus lactone against hydrogen peroxide-induced dysfunction and oxidative stress in osteoblastic MC3T3-E1 cells. Toxicol In Vitro 23: 862-867, 2009.

34. Yan XT, Lee SH, Li W, Sun YN, Yang SY, Jang HD and Kim YH: Evaluation of the antioxidant and anti-osteoporosis activities of chemical constituents of the fruits of Prunus mume. Food Chem 156: 408-415, 2014.

35. Michihara S, Tanaka T, Uzawa Y, Moriyama T and Kawamura Y: Puerarin exerted anti-osteoporotic action independent of estrogen receptor-mediated pathway. J Nutr Sci Vitaminol (Tokyo) 58: 202-209, 2012.

36. Kim H: Neuroprotective herbs for stroke therapy in traditional eastern medicine. Neurol Res 27: 287-301, 2005.

37. Wang Q, Wu T, Chen X, Ni J, Duan X, Zheng J, Qiao J, Zhou L and Wei J: Puerarin injection for unstable angina pectoris. Cochrane Database Syst Rev 3: CD004196, 2006.

38. Hwang YP and Jeong HG: Mechanism of phytoestrogen puerarin-mediated cytoprotection following oxidative injury: Estrogen receptor-dependent up-regulation of PI3K/Akt and HO-1. Toxicol Appl Pharmacol 233: 371-381, 2008.

39. Zhang MY, Qiang H, Yang HQ, Dang XQ and Wang KZ: In vitro and in vivo effects of puerarin on promotion of osteoblast bone formation. Chin J Integr Med 18: 276-282, 2012.

40. Urasopon N,Hamada Y,Cherdshewasart W and Malaivijitnond S: Preventive effects of Pueraria mirifica on bone loss in ovariectomized rats. Maturitas 59: 137-148, 2008.

41. Wong R and Rabie B: Effect of puerarin on bone formation. Osteoarthritis Cartilage 15: 894-899, 2007.

42. Tiyasatkulkovit W, Charoenphandhu N, Wongdee K, Thongbunchoo J, Krishnamra $\mathrm{N}$ and Malaivijitnond S: Upregulation of osteoblastic differentiation marker mRNA expression in osteoblast-like UMR106 cells by puerarin and phytoestrogens from Pueraria mirifica. Phytomedicine 19: $1147-1155,2012$.

43. Cao XH, Wang AH, Wang CL, Mao DZ, Lu MF, Cui YQ and Jiao RZ: Surfactin induces apoptosis in human breast cancer MCF-7 cells through a ROS/JNK-mediated mitochondrial/caspase pathway. Chem Biol Interact 183: 357-362, 2010.

44. Chipuk JE and Green DR: How do BCL-2 proteins induce mitochondrial outer membrane permeabilization? Trends Cell Biol 18: 157-164, 2008.

45. Shi RL and Zhang JJ: Protective effect of puerarin on vascular endothelial cell apoptosis induced by chemical hypoxia in vitro. Yao Xue Xue Bao 38: 103-107, 2003 (In Chinese).

46. Zhu LH, Wang L, Wang D, Jiang H, Tang QZ, Yan L, Bian ZY, Wang XA and Li H: Puerarin attenuates high-glucose-and diabetes-induced vascular smooth muscle cell proliferation by blocking PKCbeta2/Rac1-dependent signaling. Free Radic Biol Med 48: 471-482, 2010

47. Han JQ, Yu KY and He M: Effects of puerarin on the neurocyte apoptosis and p-Akt (Ser473) expressions in rats with cerebral ischemia/reperfusion injury. Zhongguo Zhong Xi Yi Jie He Za Zhi 32: 1069-1072, 2012 (In Chinese).

48. Gu YX, Du J, Si MS, Mo JJ, Qiao SC and Lai HC: The roles of PI3K/Akt signaling pathway in regulating MC3T3-E1 preosteoblast proliferation and differentiation on SLA and SLActive titanium surfaces. J Biomed Mater Res A 101: 748-754, 2013.

49. Widłak P: The DFF40/CAD endonuclease and its role in apoptosis. Acta Biochim Pol 47: 1037-1044, 2000.

50. Chua CC, Chua BH, Chen Z, Landy C and Hamdy RC: Dexamethasone induces caspase activation in murine osteoblastic MC3T3-E1 cells. Biochim Biophys Acta 1642: 79-85, 2003. 
51. Xie H, Tang LL, Luo XH, Wu XY, Wu XP, Zhou HD, Yuan LQ and Liao EY: Suppressive effect of dexamethasone on TIMP-1 production involves murine osteoblastic MC3T3-E1 cell apoptosis. Amino Acids 38: 1145-1153, 2010.

52. Quintavalle C, Brenca M, De Micco F, et al: In vivo and in vitro assessment of pathways involved in contrast media-induced renal cells apoptosis. Cell Death Dis 2: e155, 2011.

53. Liu Y, Porta A, Peng X, Gengaro K, Cunningham EB, Li H, Dominguez LA, Bellido T and Christakos S: Prevention of glucocorticoid-induced apoptosis in osteocytes and osteoblasts by calbindin-D28k. J Bone Miner Res 19: 479-490, 2004.

54. Bost F, Aouadi M, Caron L and Binétruy B: The role of MAPKs in adipocyte differentiation and obesity. Biochimie 87: 51-56, 2005.

55. Matsuguchi T, Chiba N, Bandow K, Kakimoto K, Masuda A and Ohnishi T: JNK activity is essential for Atf4 expression and late-stage osteoblast differentiation. J Bone Miner Res 24: 398-410, 2009.

56. Kim SW, Her SJ, Park SJ, Kim D, Park KS, Lee HK, Han BH, Kim MS, Shin CS and Kim SY: Ghrelin stimulates proliferation and differentiation and inhibits apoptosis in osteoblastic MC3T3-E1 cells. Bone 37: 359-369, 2005.
57. Xie H, Tang SY, Li H, Luo XH, Yuan LQ, Wang D and Liao EY: L-carnitine protects against apoptosis of murine MC3T3-E1 osteoblastic cells. Amino Acids 35: 419-423, 2008.

58. Díaz-Montero CM, Wygant JN and McIntyre BW: PI3-K/Akt-mediated anoikis resistance of human osteosarcoma cells requires Src activation. Eur J Cancer 42: 1491-1500, 2006.

59. Kim BJ, Lee YS, Lee SY, et al: Afamin secreted from nonresorbing osteoclasts acts as a chemokine for preosteoblasts via the Akt-signaling pathway. Bone 51: 431-440, 2012.

60. Cantrell DA: Phosphoinositide 3-kinase signalling pathways. J Cell Sci 114: 1439-1445, 2001.

61. Kitase Y, Barragan L, Qing H, Kondoh S, Jiang JX, Johnson ML and Bonewald LF: Mechanical induction of PGE2 in osteocytes blocks glucocorticoid-induced apoptosis through both the $\beta$-catenin and PKA pathways. J Bone Miner Res 25: 2657-2668, 2010 . 